\title{
Synthesis and characterization of a novel cationic polymer gene delivery vector
}

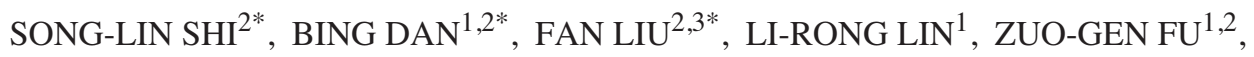 \\ GUANG-JUN JING ${ }^{3}$, TIAN-CI YANG ${ }^{1}$ and ZHONG-YING ZHANG ${ }^{1}$ \\ ${ }^{1}$ Center of Clinical Laboratory, Zhongshan Hospital, Medical College of Xiamen University, Xiamen 361004; \\ ${ }^{2}$ Medical College of Xiamen University, Xiamen $361005 ;{ }^{3}$ School of Life Sciences, \\ Xiamen University, Xiamen 361005, P.R. China
}

Received April 26, 2010; Accepted June 29, 2010

DOI: 10.3892/ijmm_00000490

\begin{abstract}
Non-viral vectors have been widely used in gene transfection. However, its drawbacks limit its applications. In this study, a novel cationic polymer was developed as a DNA condensing agent for systemic gene delivery. Its transfection efficiency, cytotoxicity, and biocompatibility were also evaluated. Sofast, novel cationic polymer of branched polyethlenimine, was constructed by chemical methods. Its diameter, $\zeta$ potential, nucleic acid binding ability, and anti-nuclease ability were detected by electron microscopy and gel electrophoresis. In vitro, the efficiency of transfection was measured by comparing it with other gene vectors in different cell lines. MTT assay was performed to determine cytotoxicity. The compatibility of Sofast gene vector in the serum and its stability were investigated. Mouse, guinea pig and rabbit were used to process the toxic, allergenic, and pyrogenic properties of the vector in vivo. The in vivo expression was performed in the guinea pig. The results from an in vitro assay proved that the Sofast gene vector had a higher transfection efficiency than other gene vectors in a variety of primary cell cultures and transformed cell lines. The cytotoxicity assay showed a lower cytotoxicity and the cellular survival rate was $>90 \%$. The Sofast gene vector possessed compatibility with the serum and was fit to be transported at normal temperature. The results from in vivo tests indicated that the Sofast gene vector had greatly lower cytotoxicity, better biocompatibility, and higher transfection efficiency compared with other gene vectors. Because the Sofast gene vector had higher transfection efficiency, lower cytotoxicity and better compatibility than other gene vectors, it could be used for gene transfection both in vitro and in vivo.
\end{abstract}

Correspondence to: Dr Tian-Ci Yang or Dr Zhong-Ying Zhang, Center of Clinical Laboratory, Zhongshan Hospital, Medical College of Xiamen University, Xiamen 361004, P.R. China

E-mail: ytc@xmu.edu.cn

E-mail: zhangzy1121@xmu.edu.cn

"Contributed equally

Key words: cationic polymer, gene delivery, transfection, non-viral vector

\section{Introduction}

Presently, viral and non-viral vectors are the leading types of gene vectors each possessing advantages and disadvantages. Viral gene vectors have higher transfection efficiency but at present a deficiency in biosafety. Non-viral gene vectors, although having a lower transfection efficiency than viral gene vectors, are receiving increasingly more attention for their considerable merits, including low toxicity and immunogenicity, easy mass production, and low cost. At present, nonviral vectors with the most potential are cationic liposomes and cationic polymers (1-7).

The cationic polymer recruits DNA through electrostatic adsorption and forms a nanometer-scale polyelectrolyte complex, which enters the cell by endocytosis and protects genes from anti-nuclease. Water-soluble cationic polymers form stable polyelectrolyte complexes by condensing DNA (8) and penetrate the membrane of the receptor by simulating the virus structure before finally was degrading. Research on non-viral gene vectors focused on cationic polymer due to its non-carcinogenic properties. When compared with a viral gene vector, the non-viral vector has a lot of advantages, but its low efficiency of transfection, weak degradability, and incompatibility constrains its use (9).

In this study, a cationic polymer named Sofast gene vector was constructed. It had an average molecular weight of $30 \mathrm{kD}$ and a branch structure. It was used to transfer macromolecular nucleic acid both in vivo and in vitro. A series of tests were performed to analyze transfection efficiency, cytotoxicity, compatibility with the serum, stability of physicochemical properties, and biocompatibility. These tests verified that the Sofast gene vector can be used in gene transfection both in vitro and in vivo because of its high transfection efficiency, low cytotoxicity, and good cellular compatibility. This research serves as the foundation for further study into the clinical and experimental use of the gene vector.

\section{Materials and methods}

Materials and reagents. Chemicals and cell culture medium, Aziridine, 2,2'-Azobis(2-methylpropionamidine) dihydrochloride, diethanolamine, and 4-dimethylamine pyridine were purchased from Guangzhou Diecheng Trading Co. (China). 
Sulfuric acid, ethane, ethyl acetate, dimethyl sulfoxide (DMSO), and glycerol were purchased from Ximen Luyin Co. (China). Silica gel column was purchased from Qingdao Gaonengda Chemical Co. (China). Cell culture media Optiminimum essential media (Opti-MEM), Dulbecco's Modified Eagle's Medium (DMEM), and Eosin Methylene Blue Agar (EMB) were purchased from Gibco (USA).

Plasmid. pEGFPN1 and pCMV-lacZ were purchased from Clontech Co. (USA). pGL3 was purchased from Promega (USA).

Transfect reagents. Lipofectamine 2000 was purchased from Invitrogen (USA). Cytopure and Jet PEI were purchased from Qbiogene (USA). Superfect was purchased from Qiagen (USA).

Cell lines and animals. Human embryonic kidney 293 cells (HEK293 cells) and human umbilical vein epithelial cells (HUVECs) were purchased from American Type Culture Collection (ATCC). Guinea pigs were from Xiamen University Laboratory Animal Center (XMULAC). All animal studies were approved by the Review Board, Medical College of Xiamen University.

Construction of cationic polymer gene transfection vector. After cyclopolymerization, the aziridine monomer was made into a cationic polymer with a molecular weight of $30 \mathrm{kD}$ and a branch structure catalyzed by acid. The detailed process is described in the published patent (10). The procedure involved mixing $200 \mathrm{ml}$ diluted water and $30 \mathrm{ml} 18.4 \mathrm{M}$ sulfuric acid. The reaction temperature was kept at $90^{\circ} \mathrm{C}$ as $4 \mathrm{ml} 1 \%$ aziridine was slowly added. Then $0.4 \mathrm{~g}$ 2,2'-azobis(2-methylpropionamidine) dihydrochloride as initiator, $90 \mathrm{ml}$ diethanolamine, and $15 \mathrm{~g}$ 4-dimethylamine pyridine were added under nitrogen. The reaction system was kept at $90^{\circ} \mathrm{C}$ for $4 \mathrm{~h}$. After it was cooled down to room temperature and concentrated under a reduced pressure condition, the mixture was purified with silica gel column chromatography. The reaction mixture was applied to a silica gel column $(60-100$ mesh, 30x300 mm) and ethane. Ethyl acetate $(3: 1, v / v)$ was used as mobile phase. The fractions containing cationic polymer were collected and vacuum dried. The average molecular weight of the cationic polymer was determined by high performance gel chromatography according to the method described by Zhang (11). Cationic polymer $(50 \mathrm{mg})$ was dissolved in $10 \mathrm{ml}$ gene vector solution $\left(0.45 \% \mathrm{NaCl}, 49 \% \mathrm{DMSO}\right.$, and $1 \%$ glycerol) at $56^{\circ} \mathrm{C}$ for a final concentration of $5 \mathrm{mg} / \mathrm{ml}$. After filtering with $0.2 \mu \mathrm{m}$ membrane filter (Bandao Industry Co. China), the mixture, named Sofast gene vector, was used for in vitro gene transfection. Another $50 \mathrm{mg}$ cationic polymer was dissolved in $10 \mathrm{ml}$ gene vector solution $(4.95 \%$ glucose and $1 \%$ glycerol) at $56^{\circ} \mathrm{C}$ for a final concentration of $5 \mathrm{mg} / \mathrm{ml}$. After filtering with $0.2 \mu \mathrm{m}$ membrane filter, the mixture was used for in vivo gene transfection.

Analysis of Sofast gene vector in vitro. All studies were performed in triplicate or were repeated three different times.

Diameter and $\zeta$ potential of Sofast/DNA complex. Sofast gene vector $(5 \mathrm{mg} / \mathrm{ml})(0,4,8,16,32,64,128$, and $256 \mu \mathrm{l})$ were diluted in Opti-MEM culture medium, and plasmid DNA (pEGFPN1, $20 \mu \mathrm{g} / \mathrm{ml}$ ) was added until the mass ratio of Sofast gene vector to DNA was $0: 1,0.5: 1,1: 1,2: 1,4: 1,8: 1$, 16:1, and 32:1, respectively. The Sofast/DNA complexes were made with a final volume of 4,000 $\mu 1$. Then, they were incubated at room temperature for $15 \mathrm{~min}$. The complexes dropped on the carbon coating copper mesh were stained with uranyl acetate for $30 \mathrm{sec}$ and dried in a vacuum desiccator for $3 \mathrm{~h}$. The configuration and size of the complex were observed using transmission electron microscopy (TEM). The diameter and $\zeta$ potential were determined by laser particle sizer (LPS) while distribution was analyzed by automatic mode.

Anti-nuclease assay of Sofast/DNA complex. The Sofast/DNA solution containing $2 \mu \mathrm{g}$ plasmid was made with the mass ratio of $0: 1,4: 1,8: 1,16: 1$, and 32:1 at a final volume of $200 \mu 1$. After incubating at room temperature for $15 \mathrm{~min}$, Sofast/ pEGFPN1 was mixed with $50 \mu 1$ nuclease I $(100 \mathrm{U} / \mathrm{ml})$ and incubated again at $37^{\circ} \mathrm{C}$ for $90 \mathrm{~min}$. Next, $100 \mu \mathrm{l}$ stop solution (pH $8.0400 \mathrm{mmol} \mathrm{NaCl}$ and $100 \mathrm{mmol}$ EDTA) was added, and the mixture was kept at room temperature for $5 \mathrm{~min}$. After that, the mixture was incubated at $65^{\circ} \mathrm{C}$ overnight with $12 \mu 110 \%$ SDS. DNA was dissociated from the complex. The $10-\mu 1$ solution was analyzed with $0.8 \%$ agarose gel for $30 \mathrm{~min}$ at $100 \mathrm{~V}$. Undigested pEGFPN1 was used as control.

Nucleotide binding capacity of Sofast gene vector. Plasmid DNA, pEGFPN1, pCMV-lacZ, and pGL3 (20 $\mu \mathrm{g}$ each) were diluted in Opti-MEM, separately. The mass ratio of Sofast gene vector to DNA was $0: 1,0.5: 1,1: 1,2: 1,4: 1,8: 1,16: 1$, and $32: 1$. After incubating at room temperature for $15 \mathrm{~min}$, $15 \mu 1$ samples were detected by agarose gel electrophoresis and visualized under UV light.

\section{Gene transfection assay with Sofast gene vector in vitro}

Cell culture. HEK293 cells were cultured in DMEM with $10 \%$ fetal bovine serum, $100 \mathrm{U} / \mathrm{ml}$ penicillin and $100 \mathrm{U} / \mathrm{ml}$ streptomycin. HUVECs cells were cultured in EMB medium containing serum and multiple growth factors. Both cell lines were cultured at $37^{\circ} \mathrm{C}$ and $5 \% \mathrm{CO}_{2}$.

Gene transfection. HEK293 cells were seeded in 24-well culture dishes at a concentration of $4.0 \times 10^{5}$ cells $/ \mathrm{ml}$ for $16-24 \mathrm{~h}$. The complex, which was made of $0.6 \mu \mathrm{g}$ plasmid DNA (pEGFPN1, pCMV-lacZ, and pGL3) and Sofast gene vector with a final volume of $60 \mu 1$, was incubated at room temperature for $15 \mathrm{~min}$. Other gene vectors (Lipofectamine 2000 , Jet PEI, and Superfect) were used following the constructions. Cells were cultured with $60 \mu 1$ of the complex at $37^{\circ} \mathrm{C}$ for $48 \mathrm{~h}$ and $5 \% \mathrm{CO}_{2}$.

Analysis of expression of report genes. The green fluorescence signal of GFP was detected by fluorescence microscopy (Olympus 1x70, Japan). The percentage of cells with positive green light was counted based on 100 cells in three different eyeshots. Luciferase activity was detected according to the instructions provided by Promega Company. Cells were washed with PBS and shaken with $150 \mu 1$ lysis buffer for $10 \mathrm{~min}$. After centrifugation, $6 \mu 1$ supernatant was used for the determination of protein concentration. Another $10-\mu 1$ supernatant solutions were used to measure luciferase activity (RLU/mg). For $\beta$-galactosidase cells were fixed in 
4\% paraformaldehyde for $5 \mathrm{~min}$ and then stained in $\mathrm{X}$-gal solution at $37^{\circ} \mathrm{C}$ for $3 \mathrm{~h}$. The percentage of cells with navy blue stain was counted based on 100 cells in three different eyeshots. For gene transfer efficiency of special cell line, HUVECs were employed for further determination of transfection effect of Sofast gene vectors on cells that were usually unaffected by transfection. Sofast, Jet PEI, Superfect, and Lipofectamine 2000 were used to tansfect pEGFPN1 into HUVECs according to the protocol for the transfection of HEK293, but the mass ratio of gene vector to DNA was decreased by $50 \%$.

Cytotoxicity assay of Sofast gene vector. Four different gene vectors, Sofast, Superfect, Lipofectamine 2000, and Jet PEI, were each mixed with $0.15 \mu \mathrm{g}$ plasmid DNA (pEGFPN1) to form gene vector/DNA complexes. The mass ratio of Sofast, Superfect, and Lipofectamine 2000 gene vector to DNA was 16,15 , and 5 , respectively, and the N/P ratio of Jet PEI gene vector to DNA was 10. These gene vector/DNA complexes were used to transfect HEK293 cells. Transfected cells were cultured for $48 \mathrm{~h}$ and then harvested to determine the survival rate through MTT assay. The untreated HEK293 cells were taken as control.

Compatibility with serum of Sofast gene vector. Different transfection procedures were employed to determine the effect of serum on the transfection efficiency of the Sofast gene vector based on the evaluation of luciferase activity in HEK293 cells transfected with pGL3 plasmid using the Sofast gene vector. Cells were seeded at a density of 40-50\%. After washing with PBS, cells in the traditional procedure group (controls) were cultured in serum-free medium with Sofast/ DNA complex for $4 \mathrm{~h}$, and then cultured in medium containing $10 \%$ serum for another 24-48 h. Experiment group cells were cultured in the medium containing $10 \%$ serum and transfected with Sofast gene vector without changing the medium.

Stability analysis of Sofast gene vector. pEGFPN1 plasmid was used to transfect HEK293 cells to determine the stability of Sofast gene vector. The vector was stored at $4^{\circ} \mathrm{C}$ for 1 46 months (two-month intervals) or at room temperature for 30 days before use. The transfection efficiency of Sofast gene vector stored for different periods at different temperatures was investigated.

\section{Analysis of Sofast gene vector in vivo}

Analysis of toxic, allergenic, and pyrogenic properties. For acute toxicity assay ten male mice with a body weight of $18-22 \mathrm{~g}$ each were tail mainlined with $0.5 \mathrm{ml} 5 \mathrm{mg} / \mathrm{ml}$ Sofast/ pEGFPN1 complexes and observed for $48 \mathrm{~h}$ to verify any death or adverse reaction. Two mice injected with normal saline were used as negative control.

For sub-acute and chronic toxicity assay the protocol was similar with that of the acute assay except that Sofast/ pEGFPN1 was given at a moderate dose. Mice were injected intravenously with $0.5 \mathrm{ml}$ of $5 \mathrm{mg} / \mathrm{ml}$ Sofast/pEGFPN1 complexes and observed for 8 weeks and 5 months for subacute and chronic toxicity assay. Two mice injected with normal saline were used as negative controls.

For allergy assay, six guinea pigs with a body weight of 250-350 g each were injected intraperitoneally with $0.5 \mathrm{ml}$
$0.5 \mathrm{mg} / \mathrm{ml}$ Sofast/pEGFPN1 complexes every other day for 3 times a day. They were then divided into two groups. One group was injected intrajugularly with $1 \mathrm{ml} 0.5 \mathrm{mg} / \mathrm{ml}$ Sofast/ pEGFPN1 complexes on the 14th day after the first injection. Another group was injected on the 21 st day after the first injection. One group was injected with normal saline and used as negative control.

For the pyrogen test three rabbits with a body weight of $2.5 \mathrm{~kg}$ each were used for the pyrogen test. The average anus temperature was counted from 3 repeats. Body temperature was measured after the rabbits were injected with $2.5 \mathrm{ml}$ $5 \mathrm{mg} / \mathrm{ml} \mathrm{Sofast} / \mathrm{DNA}$ complexes $(1 \mathrm{ml} / \mathrm{kg})$ for 1,2 , and $3 \mathrm{~h}$. One rabbit injected with normal saline was used as negative control.

In vivo application: the expression of GFP gene in lung, liver, and kidney cells. Jet PEI (linear PEI $22 \mathrm{kDa}$ ) is chemically closer to Sofast and has been reported to result in high lung expression (4,12). Lipofectamine 2000 is a cationic liposome vector that provides high transfection efficiency and high levels of transgenic expression in a range of mammalian cell types in vitro (13). This study compared the transfection efficiency of Sofast with Jet PEI and Lipofectamine 2000 in two separate experiments. In the comparison of Sofast and Jet PEI, 15 male guinea pigs, each eight-week-old and weighing 250-300 g, were randomly divided into three groups, A, Sofast/pEGFPN1; B, Jet PEI/pEGFPN1; and C, negative control (pEGFPN1). pEGFPN1 $(50 \mu \mathrm{g})$ plasmid and $75 \mu \mathrm{l}$ Sofast gene vector were each diluted in $2 \mathrm{ml} \mathrm{5 \%}$ glucose solution. The Sofast diluents were then dropped into pEGFPN1 plasmid diluents and incubated at room temperature for 15 min. Jet PEI was diluted in $2 \mathrm{ml} \mathrm{5 \%}$ glucose solution according to the instructions. The mice in groups A and B were injected intravenously in the tail with $200 \mu 1$ Sofast/pEGFPN1 and Jet PEI/pEGFPN1 complexes, respectively. The negative group C was injected with $200 \mu 1$ pEGFPN1 diluents. In the comparative experiment of Sofast and Lipofectamine 2000, Lipofectamine 2000 was used instead of Jet PEI in group B. After $48 \mathrm{~h}$ of transfection, the tail, lung, liver, and kidney were stained and observed according to the routine tissue section protocol. The frozen sections of parts of the tail, lung, liver, and kidney were blazed with blue light and observed under a fluorescence microscope. Flow cytometry (FCM) was performed to investigate the percentages and fluorescence intensity of positive cells. Data were analyzed with SPSS 13.0 software and the results are shown with mean \pm standard deviation.

\section{Results}

\section{Analyses of Sofast gene vector in vitro}

Formation, diameter, and $\zeta$ potential of Sofast/DNA complexes. The average molecular weight of the cationic polymer determined by high performance gel chromatography was $\sim 30 \mathrm{kD}$. The surface of the cationic polymer was surrounded with positive charges while plasmid DNA had negative charges from its phosphate. Cationic polymer was combined with plasmid DNA to form complexes through electrostatic interaction. The diameter of Sofast/DNA complexes gradually decreased along with the increase in the mass ratio of Sofast 


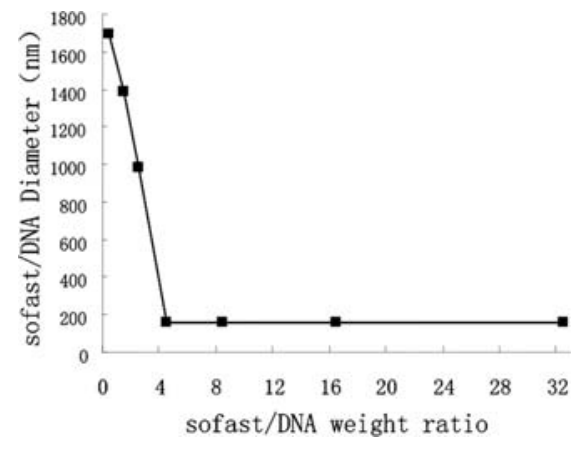

Figure 1. Particle size of Sofast/DNA complex with different Sofast/DNA weight ratio.

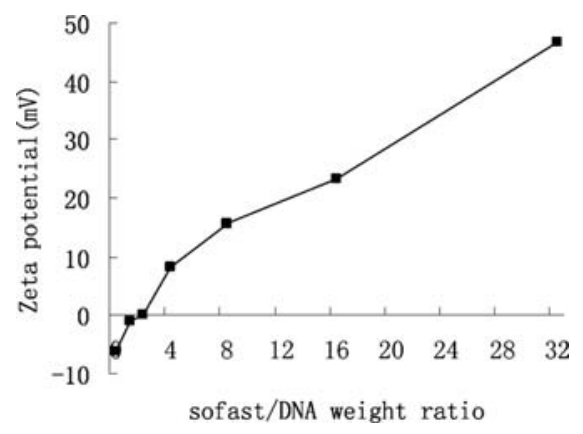

Figure 2. $\zeta$ potential of Sofast/DNA complex with different Sofast/DNA weight ratio.

gene vector to DNA. When the mass ratio of Sofast gene vector to DNA was greater $\geq 4: 1$ threshold, the Sofast gene vector was able to condense the DNA to form the complexes, whose diameter varied from 154.8 to $162.7 \mathrm{~nm}$ (Fig. 1). The $\zeta$ potential of Sofast/DNA complexes increased with the enhancement of the mass ratio of Sofast gene vector to DNA, which ranged from -6.2 to $+46.6 \mathrm{mV}$ (Fig. 2). The results from TEM indicate that the compressed Sofast/DNA complexes were pellet-shaped with an average diameter of $160 \mathrm{~nm}$, which was consistent with the data from LPS (Fig. 3).

Anti-nuclease ability of Sofast/DNA complexes. After being digested with nuclease I at $37^{\circ} \mathrm{C}$ for $90 \mathrm{~min}$, the Sofast/ pEGFPN1 was subjected to DNA electrophoresis. The results indicate that the naked DNA, whose mass ratio of Sofast gene vector to DNA was 0:1, was degraded. However, the plasmid DNA kept its integrity when the mass ratio of Sofast gene vector to DNA was $4: 1,8: 1,16: 1$, and $32: 1$, suggesting that the vector could protect plasmid DNA from the digestion of nuclease I (Fig. 4).

DNA retardation assay. DNA retardation assay was used to test the binding capacity of gene vector to DNA. The results (Fig. 5) show a typical plasmid band presented without the Sofast gene vector. When the mass ratio of Sofast gene vector to DNA was $0.5: 1$, the electrophoretic velocity of plasmid DNA was delayed, and only an obscure DNA trail was observed. When the mass ratio was up to 1 , only parts of the

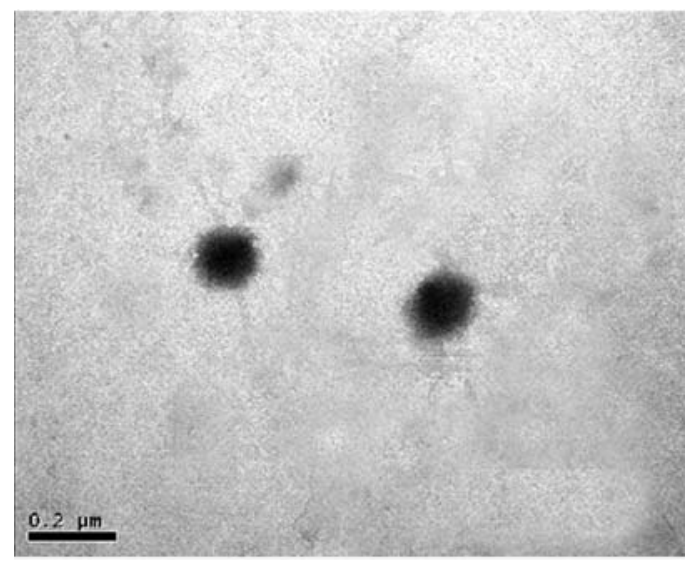

Figure 3. Transmission electron microscope profile of Sofast/DNA complex with ratio of $4: 1$; scale indicates $200 \mathrm{~nm}$.

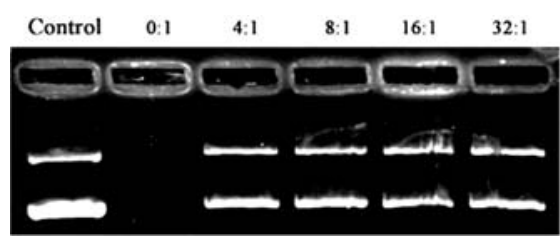

Figure 4. Stability of Sofast/DNA complexes to DNase I at various weight ratios (w/w). Numbers $0: 1,4: 1,8: 1,16: 1$, and $32: 1$ indicate weight ratio of Sofast and DNA.

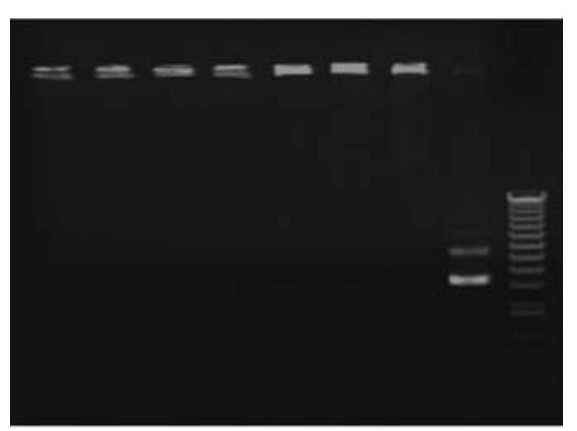

Figure 5. Gel electrophoresis of sofast/pEGFPN1 complexes. The numbers $0,0.5,1,2,4,8,16$, and 32 indicate the weight ratio of sofast and DNA. 0 , no Sofast gene carrier; M, DNA marker.

undifferentiated DNA moved out of the spotting hole. When the ratio was $>2$, the entire DNA bound with Sofast gene vector. The results of DNA retardation assay for $\mathrm{pCMV-lacZ}$ and pGL3 plasmids were similar with that of pEGFPN1. These results prove that cationic polymer Sofast gene vector possessed high DNA binding capacity.

\section{Analyses of transfection efficiency in vitro}

Expression of report gene of GFP. The positive cells transfected with Sofast gene vector accounted for $\sim 60 \%$ of the total. The fluorescence signal was stronger, suggesting high efficiency of transfection, while the transfection rates of other gene vectors varied from 50 to $55 \%$, and their fluorescence 


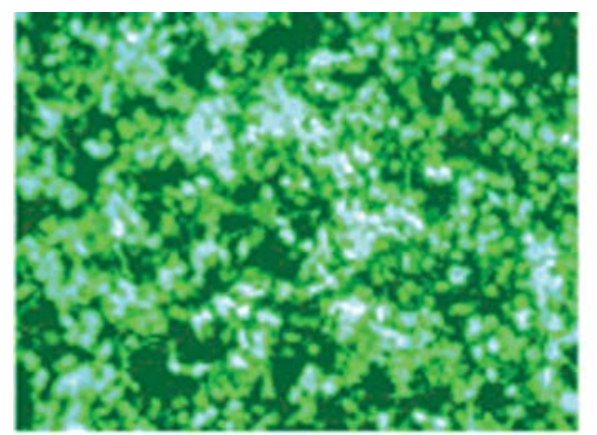

Sofast

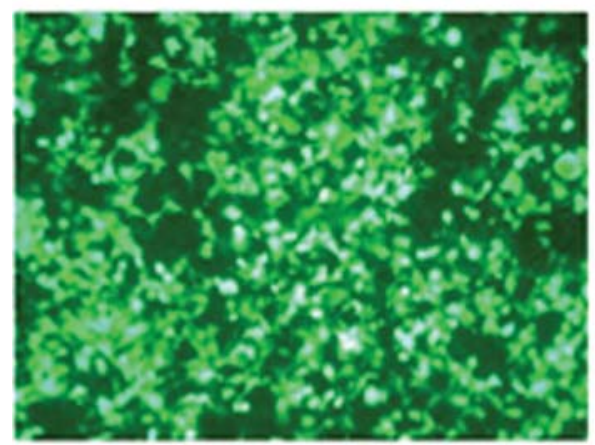

Superfect

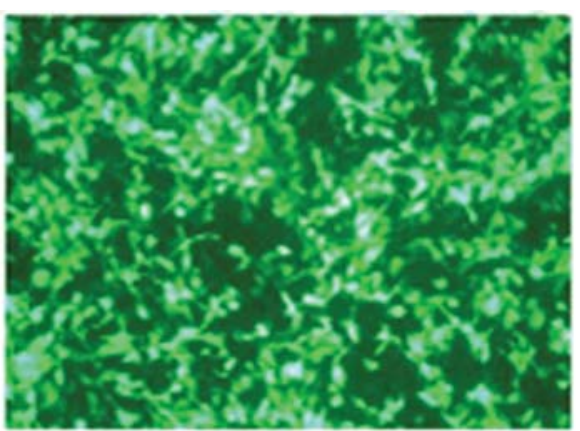

Jet PEI

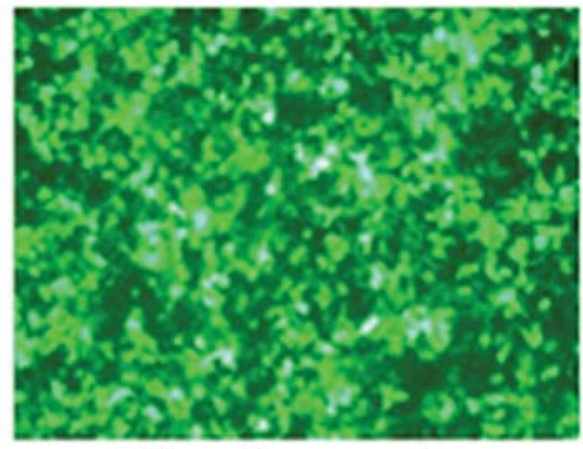

Lipofectamine

Figure 6. Typical picture of GFP gene transfection by different gene carriers with HEK293.

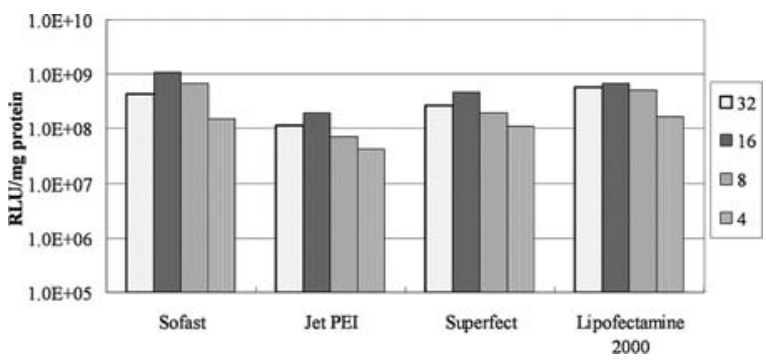

Figure 7. Luciferase gene transfection efficiency by using different gene carriers with HEK293 cells. Weight ratio (w/w) Sofast and DNA, 32, 16, 8, and 4. Superfect gene carrier w/w, 30, 15, 7.5, and 3.75. Lipofectamine 2000 $\mathrm{w} / \mathrm{w}, 10,5,2.5$, and 1.25 . The number N/P of Jet PEI is $20,10,5$, and 2.5 .

signal was weak (Fig. 6). The percentage of positive cells transfected with Sofast gene vector and the expression level of GFP were high.

Activity of luciferase. The activity of luciferase expressed by the Sofast-transfected pGL3 plasmid resulted in the highest transfection efficiency. When the mass ratio of Sofast to DNA was $16: 1$, the luciferase activity of transfected cells, with $>10^{9} \mathrm{RLU} / \mathrm{mg}$ proteins, was 5, 2.3, and 1.6 times of the cells transfected with Jet PEI, Superfect and Lipofectamine 2000, suggesting the transfection efficiency of Sofast was higher than that of the other gene vectors (Fig. 7).

$\beta$-galactosidase staining. The product of LacZ gene expression was $\beta$-galactosidase, which can catalyze the hydrolysis of X-gal. The hydrolysis product exhibited a blue color. After being transfected with pCMV-LacZ plasmid, more cells with blue color were observed, indicating that the transfection efficiency was higher. Results show that $95 \%$ of HEK293 cells transfected with Sofast gene vector showed blue signal. Percentages of the positive cells transfected with Jet PEI, Superfect and Lipofectamine 2000 gene vectors were 75,85 , and $80 \%$, respectively (Fig. 8).

Confirmation of transfection efficiency in specific cell lines. HUVECs were a primary culture derived from the umbilical vein. Different gene vectors were used to transfect pEGFPN1 into HUVECs. The results show that the percentages of positive cells transfected with Sofast, Jet PEI, and Superfect and Lipofectamine 2000 were 20, 10, 5 and $0.05 \%$, indicating that Sofast gene vector had the highest transfection efficiency (Fig. 9). For the cytotoxicity assay, the optimum proportion between gene vector and DNA was determined by gene transfection. MTT assay was performed to investigate the survival rate of cells transfected with different gene vectors. The results show that the survival rate of cells transfected with Jet PEI, Superfect, and Lipofectamine 2000 were 91.25, 87.69 , and $83.65 \%$, respectively. However, the cell survival rate of cells transfected with Sofast gene vector was up to $94.23 \%$, indicating the lowest cytotoxicity (Fig. 10).

Compatibility with serum. Different transfection procedures were taken to transfect pGL3 plasmid with Sofast gene vector. The luciferase activity in HEK293 cells indicated that there was no significant difference between the traditional transfection procedure and the modified procedures, which suggests that Sofast gene vector had excellent compatibility with serum (Fig. 11).

Stability of Sofast gene vector. pEGFPN1 was transfected into HEK293 cells with Sofast gene vector stored at $4^{\circ} \mathrm{C}$ for 


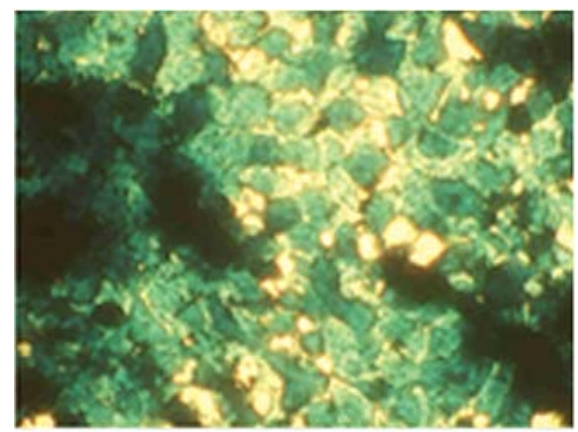

Sofast

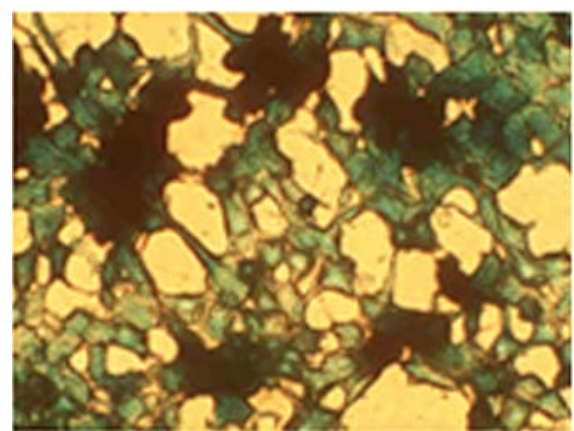

Superfect

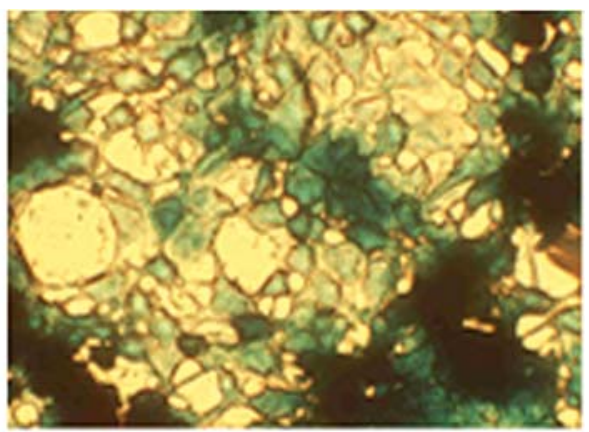

Jet PEI

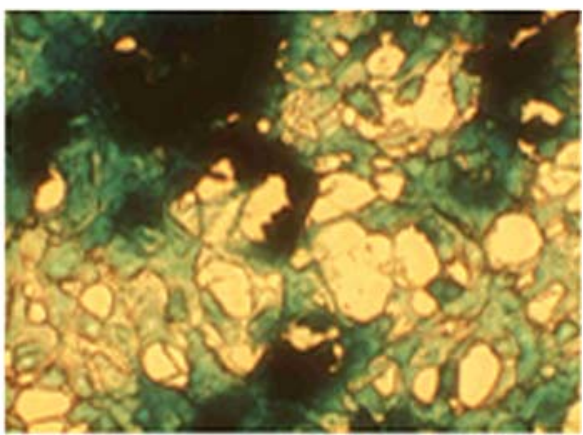

Lipofectamine

Figure 8. The typical picture of ß-galactosidase positive cells after lacZ gene transfection by using different gene carriers with HEK293.

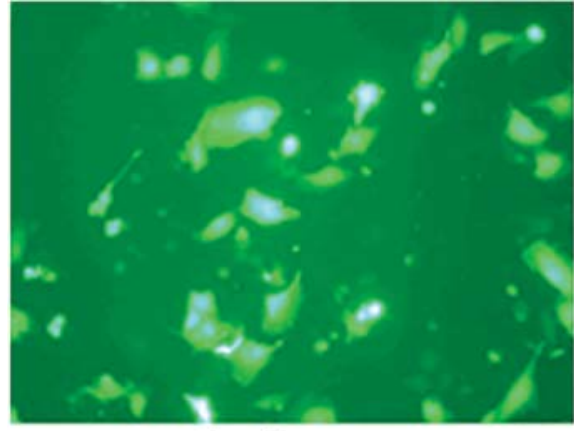

Sofast

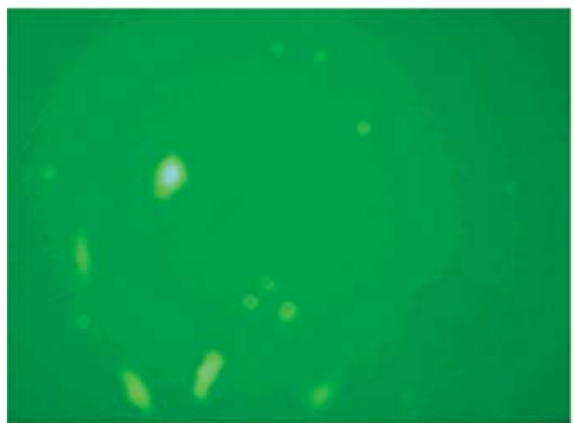

Superfect

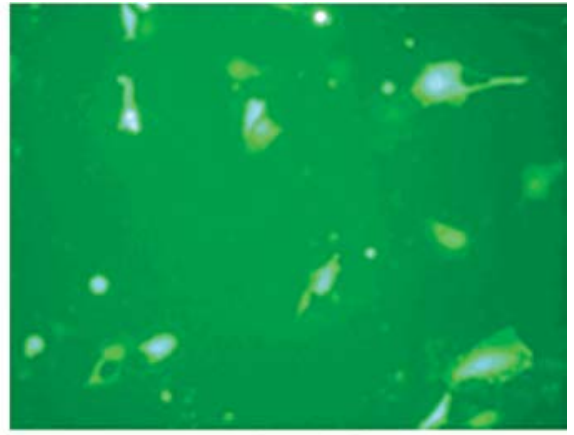

Jet PEI

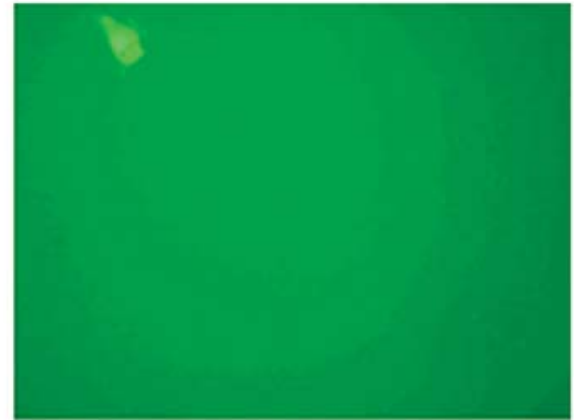

Lipofectamine

Figure 9. Typical picture of GFP gene transfection by different gene carriers with HUV-EC.

different periods (1-46 months). The results show that the efficiency of transfection were all $>90 \%$ with no significant difference. The transfection efficiency was still $>90 \%$ after storing at room temperature for 30 days. Thus, the Sofast gene vector was stable and fit be transported at a normal temperature. It can be stored for $>3$ years at $4^{\circ} \mathrm{C}$. 


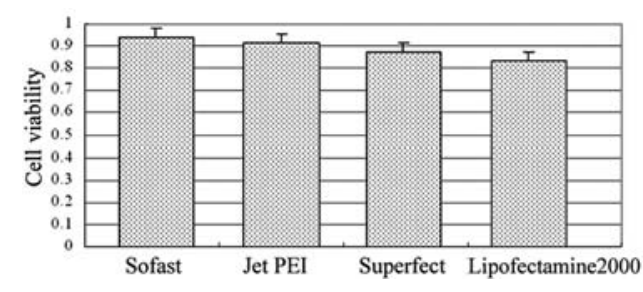

Figure 10. HEK293 cell livability by using different gene carriers.

\section{Analyses of Sofast gene vector (in vivo)}

Cytotoxicity, allergenic test, and pyrogen detection of Sofast gene vector in vivo. Acute toxicity tests indicated that the experimental group had no adverse reaction and death. It did not have any significant difference with the control group. The chronic toxicity test results show that no adverse reaction in the experimental group was observed for 8 weeks. The body weight and food and water intake remained constant up to 5 months, and there was no adverse reaction or death. The paraffin section of liver and kidney tissues did not show aberrant histological or cytological changes and had no significant difference with the control group. The average anus temperature was $38.9^{\circ} \mathrm{C}$ before mainline and $39.1^{\circ} \mathrm{C}$, $39^{\circ} \mathrm{C}$, and $39^{\circ} \mathrm{C}$ after being injected with $2.5 \mathrm{ml} 5 \mathrm{mg} / \mathrm{ml}$ Sofast/pEGFPN1 complexes for 1, 2, and $3 \mathrm{~h}$, respectively. This suggests that there was no pyrogen in the Sofast/pEGFPN1 complexes.

Expression of GFP gene in vivo. Guinea pigs were mainlined with Sofast/pEGFPN1, Jet PEI/pEGFPN1, and pEGFPN1, and cells were cultured for $48 \mathrm{~h}$. The paraffin section results show that there was no inflammatory cell infiltration in the tail, lung, liver, and kidney cells. The frozen-embedded sections of lung tissues, as well as the cortical and medullary areas of the liver and kidney in group A (Sofast/ pEGFPN1) and B (Jet PEI /pEGFPN1), were observed under a fluorescence microscope. The green fluorescence scattered in tissues and density of the signal were stronger in group A compared to group B while there was no signal detected in group C (Fig. 12). The rate and fluorescence intensity of GFP-positive cells was detected by FCM, and the results indicate that the number of GFP-positive cells and fluorescence intensity in groups A and $B$ were higher than that of group $C(p<0.01)$. The number of GFP-positive cells in the lung was higher than that of the liver and kidney. There was no significant difference in the number of GFP-positive cells between the liver and kidney $(p<0.01)$. Percentages of GFP-positive cells transfected with Sofast/pEGFPN1 in lung and kidney tissues were higher than that of cells transfected with Jet PEI/pEGFPN1. Similar changes in the fluorescence intensity were observed (Table I). Thus, the Sofast gene vector was more effective than Jet PEI when used for in vivo transfection.

In another independent comparative experiment of Sofast and Lipofectamine 2000, similar results were obtained. Observation of the frozen-embedded sections of lung tissues and the cortical and medullary areas of liver and kidney under a fluorescence microscope showed green fluorescence scattered in the tissues. The density of signal in group A (Sofast/ pEGFPN1) was stronger than in group B (Lipofectamine

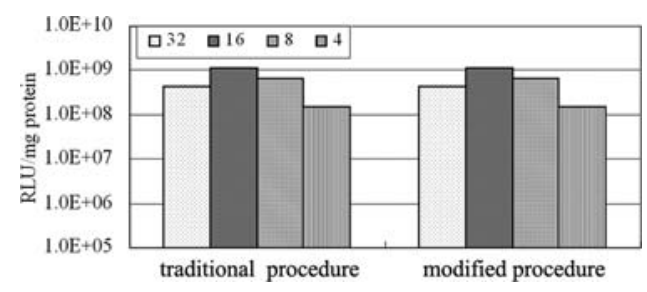

Figure 11. Luciferase gene transfection efficiency by using different transfection procedures with HEK293 cells. The numbers 32, 16, 8, and 4 indicate the weight ratio of sofast and DNA. During the traditional transfection procedure, cells were raised in the serum-free medium with Sofast/DNA complex for $4 \mathrm{~h}$, and then cultured in the medium containing $10 \%$ serum for another $24-48 \mathrm{~h}$. In the modified procedure group, cells were cultured in the medium containing $10 \%$ serum and transfected with Sofast gene vector without changing the medium. Similar transfection efficiency sugguested excellent compatibility of Sofast gene vector with serum.

2000/pEGFPN1), while there was no signal detected in group $\mathrm{C}$ (pEGFPN1) (Fig. 13). The results of FCM indicate that the number of GFP-positive cells in group A and B was higher than that in group $\mathrm{C}(\mathrm{p}<0.01)$, and the number of GFPpositive cells in the lung was higher than that in the liver and kidney. There was no significant difference in the number of GFP-positive cells between liver and kidney $(p<0.01)$. The number of GFP-positive cells of group A in liver and lung tissues was higher than that of group B. Similar changes in the fluorescence intensity were observed (Table II). These results indicate that the transfection efficiency of Sofast was more effective than that of Lipofectamine 2000 when used for in vivo transfection.

\section{Discussion}

Physical characteristics of Sofast gene vector. The Sofast cationic polymer belongs to the family of polyethlenimine [chemical structure: $\mathrm{HO}-\left(\mathrm{CH}_{2}\right)_{2}-\left(\mathrm{CH}_{2}-\mathrm{CH}_{2}-\mathrm{NH}\right)$ n- $\left(\mathrm{CH}_{2}\right)$ 2$\mathrm{OH}]$. Polyethlenimine comes in two forms, linear and branched. The branched form, which was synthesized in this research, is produced by cationic polymerization from aziridine monomers. Cationic polymer, which has high positive charge potential, can form complexes with plasmid DNA through static interactions (14). The complexes can be absorbed by the negatively-charged cellular membrane and the DNA can be transported into the cell by membrane fusion and cellular endocytosis. Midoux et al (15) reported that the complexes with diameters of 100-200 nm were easily absorbed through cytophagy. Results from the DNA retardation assay and transmission electron microscopy indicate that the Sofast gene vector completely bind DNA when the mass ratio of vector to DNA was $>2$. When the ratio ranged from 4 to 32 , the vector condensed DNA to form uniformed complexes with a diameter of $160 \mathrm{~nm}$.

$\zeta$ potential affects the efficiency of transfection under the condition of constant particle diameter. In Sofast/DNA complexes, when the mass ratio of Sofast to DNA was 4 , the surface charge rose to $+8.2 \mathrm{mV}$, and $\zeta$ potential had a positive charge. The $\zeta$ potential of the complexes kept increasing along with the enhancement of the mass ratio of Sofast to DNA. The transfection efficiency increased initially and then decreased 


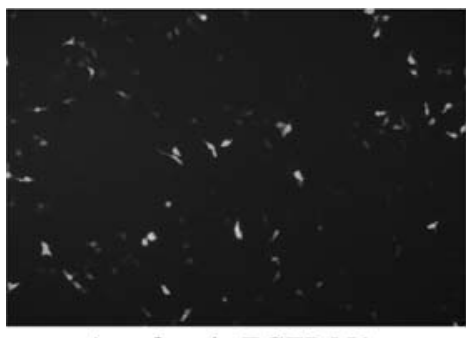

A:sofast / pEGFP N1

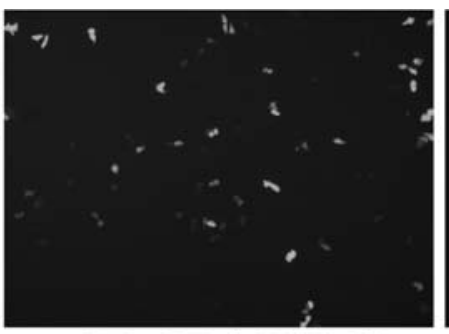

B:Jet PEI / pEGFP N1

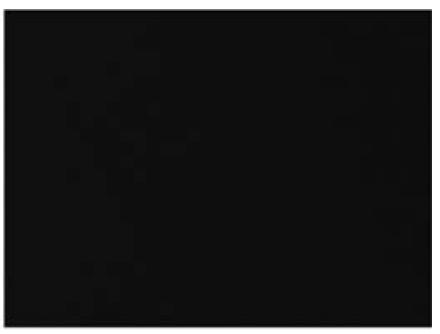

C: pEGFP N1

Figure 12. GFP expression in murine kidney tissues $48 \mathrm{~h}$ after being injected with different gene carrier/pEGFPNI complex in the comparative experiment of Sofast and Jet PEI.

Table I. Percentage and fluorescence intensity of GFP expression cells in different organs by FCM ( $\mathrm{n}=5)$.

\begin{tabular}{lrrr}
\hline & \multicolumn{2}{c}{ Test group } \\
\cline { 2 - 4 } & \multicolumn{1}{c}{ A: Sofast/pEGFPa } & B: Jet PEI/pEGFPN1 ${ }^{\mathrm{a}}$ & pEGFPN1 $^{\mathrm{a}}$ \\
\hline Liver & $8.64 \pm 1.43 / 32.1 \pm 3.8$ & $8.11 \pm 1.27 / 31.3 \pm 4.5$ & $0.34 \pm 0.09 / 2.4 \pm 1.1$ \\
Kidney & $8.22 \pm 1.34 / 38.2 \pm 4.9$ & $8.15 \pm 1.02 / 34.9 \pm 5.7$ & $0.28 \pm 0.13 / 1.4 \pm 0.8$ \\
Lung & $14.87 \pm 2.13 / 56.9 \pm 8.3$ & $13.45 \pm 1.81 / 59.0 \pm 7.1$ & $0.29 \pm 0.16 / 1.5 \pm 1.0$ \\
\hline
\end{tabular}

aPresented as precentage/fluorescence intensity.

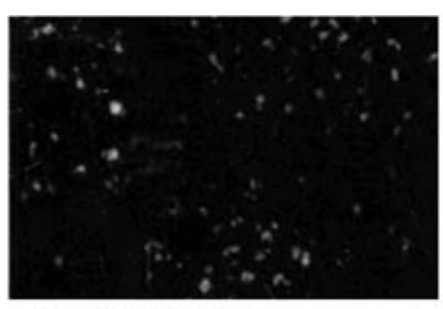

A:sofast/pEGFP N1

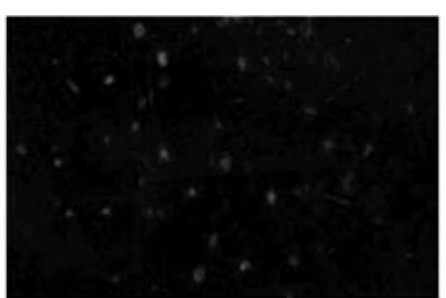

B:Lipofectamine 2000/pEGFP N1

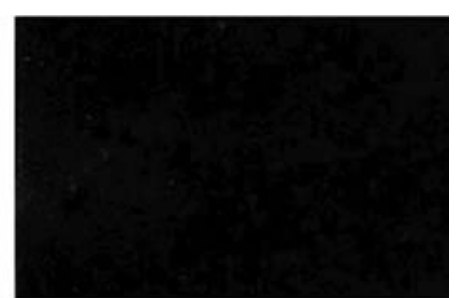

C: pEGFP N1

Figure 13. GFP expression in murine kidney tissues $48 \mathrm{~h}$ after being injected with different gene carrier/pEGFPNI complex in the comparative experiment of Sofast and Lipofectamine 2000.

Table II. Percentage and fluorescence intensity of GFP expression cells in different organs by FCM ( $\mathrm{n}=5)$.

\begin{tabular}{lrrr}
\hline & \multicolumn{3}{c}{ Test group } \\
\cline { 2 - 4 } & A: Sofast/pEGFPa & B: Lipofectamine 2000/pEGFPN1 ${ }^{\mathrm{a}}$ & C: $\mathrm{pEGFPN1 \textrm {a }}$ \\
\hline Liver & $8.52 \pm 1.54 / 32.5 \pm 3.2$ & $7.24 \pm 1.34 / 28.9 \pm 3.1$ & $0.36 \pm 0.11 / 1.5 \pm 1.0$ \\
Kidney & $7.64 \pm 1.85 / 37.2 \pm 4.2$ & $8.31 \pm 1.21 / 30.2 \pm 4.6$ & $0.33 \pm 0.29 / 0.9 \pm 0.2$ \\
Lung & $13.95 \pm 2.13 / 55.0 \pm 7.3$ & $12.62 \pm 1.81 / 41.0 \pm 6.1$ & $0.28 \pm 0.17 / 1.3 \pm 1.0$ \\
\hline
\end{tabular}

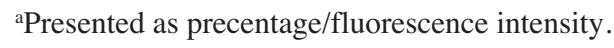

with the increase of the value of $\zeta$ potential. A summit value was finally reached when the $\zeta$ potential was $+26.4 \mathrm{mV}$ and the mass ratio of Sofast gene vector to DNA was 16 .

Anti-nuclease ability of Sofast/DNA complexes. The exogenous gene is degraded by the nuclease in the cell or blood circulation. Thus, the expression of exogenous gene in target cells limits the development of functional gene research and therapy (16). Nuclease I is a major component of nuclease in blood and cytoplasm. Furthermore, one of the important evaluation indexes for gene vectors is the protection of DNA from the degradation of nuclease $\mathrm{I}$. When the mass ratio of Sofast gene vector to DNA ranged from 4 to 32 , the vector was shown to effectively protect DNA from the degradation 
of nuclease I. It could also protect its superhelix structure, which is the precondition for functional gene expression (17).

Transfection effect of Sofast gene vector. Sofast gene vector is a novel cationic polymer. Its DNA binding capacity was first proved by DNA retardation. Three reported genes, GFP, Luciferase, and B-galactosidase, were investigated to mark the transfection efficiency of Sofast, Jet PEI, Superfect, and Lipofectamine 2000. Normally, the luciferase background activity was $10^{3}-10^{4} \mathrm{RLU} / \mathrm{mg}$. In this study, the luciferase activity of HEK293 cells transfected with all gene vectors was up to $2 \times 10^{8} \mathrm{RLU} / \mathrm{mg}$, suggesting a high transfection efficiency. The transfection efficiency of Sofast gene vector was 5, 2.3, and 1.6 times that of Jet PEI, Superfect, and Lipofectamine 2000 , respectively, suggesting that Sofast gene vector is an excellent gene vector.

Sofast gene vector has higher transfection efficiency when compared with other gene vectors. HUVECs, which are difficult to transfect with other gene vectors, was used to measure the transfection effect of the Sofast gene vector. Results indicate that the Sofast gene vectors have the highest transfection efficiency. The percentage of positive cells was $20 \%$ of the total, while the transfection efficiency of Jet PEI and Superfect was $\sim 10$ and 5\%, respectively. This indicates that the Sofast gene vector is an excellent gene vector for primary cell cultures. However, since there are so many kinds of primary cultures, the transfection efficiency of Sofast gene vectors need further investigation.

Cytotoxicity of Sofast gene vectors. Besides the transfection efficiency, cytotoxicity is also an important reference for evaluating a gene vector. The molecular weight of the cationic polymer was closely related with the cytotoxicity and transfection efficiency. The greater the molecular weight, the higher the transfection efficiency, but it is accompanied with a high cytotoxicity. In this research, an MTT assay was employed to analyze cytotoxicity. The results indicate that the survival rate of cells transfected with all the gene vectors was $>83 \%$, under an optimum dose of transfection. This suggests that the general cytotoxicity was low. Moreover, the survival rate of cells transfected with Sofast gene vector was $94.23 \%$, giving the lowest cytotoxicity result among the vectors tested.

The in vivo application of gene vectors may lead to inflammatory reaction and stimulate an immune response. The in vivo cytotoxicity test showed that no acute and chronic reaction to toxicity, allergic response, or pyrogen was found when the Sofast gene vector was used. The median lethal dose of Sofast gene vector was up to $50 \mathrm{mg} / \mathrm{ml}$, which was ten times that of the normal dose. The mice did not die, suggesting that the maximum tolerance dose was 2,500 times of that used in human subjects. It is generally thought that it could be applied for clinical use when the maximum tolerance dose in mice is 100 times of that in human subjects. The routine paraffin section showed that there was no inflammatory cell infiltration in multiviscera after injecting with Sofast/pEGFPN1 complexes for $48 \mathrm{~h}$, further proving the safety of Sofast gene vectors for in vivo application.

Compatibility of Sofast with serum. About 10-20\% bovine serum is necessary to raise cells in vitro, but this will reduce transfection efficiency. Thus, no serum is used in the routine transfection protocol to improve the transfection efficiency. This poses a problem for cells that are sensitive to serum, such as primary cells. This study compared the transfection procedure of Sofast gene vectors with routine transfection protocol. Results show that there was no significant difference between them, suggesting the perfect compatibility of Sofast gene vectors with serum. The well-prepared Sofast/DNA complexes can be directly added into cell culture. All procedures were done in a half hour. Furthermore, the culture media did not need to be replaced. As such, Sofast gene vectors had practical application advantages because of its compatibility with serum. Although the transfection of Sofast gene vector is free from the limitation of the serum, the dilution buffer for Sofast and DNA should also be serum-free, because Sofast might react with proteins of the serum before the formation of the Sofast/DNA complexes.

Application of Sofast gene vector in vivo. After mainlining, the gene vector/DNA complexes were soon deposited in the pulmonary capillary bed, most of which were intercepted by the lungs, kidneys, liver, and spleen. They were then removed by Kuffer cells in lung endothelial and hepatic sinusoidal as well as by the macrophage of the spleen (18). The observation of frozen-embedded sections of mouse liver, kidney and lung tissues showed scattered green fluorescence. Moreover, the density of green fluorescence in Sofast/ pEGFPN1 group was stronger than that of Jet PEI/pEGFPN1 group and Lipofectamine 2000/pEGFPN1 group.

In the comparison of Sofast and Jet PEI, FCM further proved that the ratio of GFP-positive cells in kidneys was the highest among the liver, kidney, and lung samples in the Sofast/pEGFPN1 group. The results in Jet PEI/pEGFPN1 group showed higher transfection efficiency in the lungs, but the number of GFP-positive cells in Sofast/pEGFPN1 group was higher than that in the Jet PEI/pEGFPN1 group. To some extent, the fluorescence intensity in different tissues was positively correlated with the percentage of the cells with GFP-expression. This indicates that the transfection efficiency of Sofast gene vector was higher than that of Jet PEI for in vivo application. This might be related with the compatibility of the cationic polymer with serum. The cationic polymer had good biocompatibility and improved the transfection efficiency for the in vivo tests. This result is in agreement with the opinion that the transfection efficiency of branched PEI is higher than that of linear PEI (19).

In the comparison of Sofast and Lipofectamine 2000, FCM proved that the number of GFP-positive cells in the lungs was highest among the liver, kidney and lung samples in the Sofast/pEGFPN1 group. Similar results were obtained in the Lipofectamine 2000/pEGFPN1 group, but the number of GFP-positive cells in Sofast/pEGFPN1 group was higher than that in the Lipofectamine 2000/pEGFPN1 group. This indicates that the transfection efficiency of Sofast gene vector was higher than Lipofectamine2000 for in vivo application.

In conclusion, there are various methods to transport genes into eukaryotic cells. The efficiency of transfection mediated by virus, including adenovirus and retrovirus is great, but low biosafety and high cost limit its broader application $(20,21)$. The non-viral vectors are receiving increasing attention for 
their easy manipulation, stability, low cost, high biosafety, and biocompatibility (22-24). However, its transfection efficiency is low and of no effect on a series of cell lines. The cationic liposome, a very popular gene vector, has shown a high transfection efficiency for in vitro tests (25). However, for in vivo applications, the cationic liposome was rapidly eliminated by serum and deposited in lung tissues (26), which can result in severe anti-inflammatory reaction and high levels of toxicity (27). These factors limited its application to some extent (28). The new generation of cationic polymeric gene vectors is getting more and more attention for its high transfection efficiency and low cytotoxicity as well as its compatibility with serum $(3,29-31)$. The Sofast gene vector constructed in this study is a novel cationic polymer. The data in this study indicate that Sofast gene vector has a lot of advantages, including high transfection efficiency, low cytotoxicity, and general applicability. The simple procedure employed and its strong biocompatibility enabled wide applications both in vivo and in vitro.

\section{Ackowledgements}

Supported by the Natural Science Foundation of Fujian Province (Grant No.: 2009J01200), the Key projects in Fujian Province Science and Technology Program (grant no. 2009D019), and the projects of Xiamen Science and Technology Program (Grant No.: 3502Z20089015).

\section{References}

1. Oliveira AC, Ferraz MP, Monteiro FJ and Simoes S: Cationic liposome-DNA complexes as gene delivery vectors: Development and behaviour towards bone-like cells. Acta Biomater 5: 2142-2151, 2009

2. Zohra FT, Chowdhury EH and Akaike T: High performance mRNA transfection through carbonate apatite-cationic liposome conjugates. Biomaterials 30: 4006-4013, 2009.

3. Gao X, Kuruba R, Damodaran K, Day BW, Liu D and Li S Polyhydroxylalkyleneamines: A class of hydrophilic cationic polymer-based gene transfer agents. J Control Release 137: 38-45, 2009.

4. Wightman L, Kircheis R, Rossler V, et al: Different behavior of branched and linear polyethylenimine for gene delivery in vitro and in vivo. J Gene Med 3: 362-372, 2001.

5. Lungwitz U, Breunig M, Blunk T and Gopferich A: Polyethylenimine-based non-viral gene delivery systems. Eur J Pharm Biopharm 60: 247-266, 2005.

6. Li D, Yu H, Huang $\mathrm{H}$, et al: FGF receptor-mediated gene delivery using ligands coupled to polyethylenimine. J Biomater Appl 22: 163-180, 2007

7. Lee BW, Chae HY, Tuyen TT, et al: A comparison of non-viral vectors for gene delivery to pancreatic B-cells: Delivering a hypoxia-inducible vascular endothelial growth factor gene to rat islets. Int J Mol Med 23: 757-762, 2009.

8. Hill IR, Garnett MC, Bignotti F and Davis SS: Determination of protection from serum nuclease activity by DNA-polyelectrolyte complexes using an electrophoretic method. Anal Biochem 291: $62-68,2001$
9. Gargouri M, Sapin A, Bouli S, Becuwe P, Merlin JL and Maincent P: Optimization of a new non-viral vector for transfection: EUDRAGUR nanoparticles for the delivery of a DNA plasmid. Technol Cancer Res Treat 8: 433-444, 2009.

10. Yang T: A cationic polymer gene transfection reagent and its preparation technology. Patent no.: ZL200710008488.2, 2009.

11. Zhang ZQ: Determination of molecular weight and its distribution of polyethylenimine by high performance gel chromatography. Prog Modern Biomed 8: 356-357, 2008.

12. Zou SM, Erbacher P, Remy JS and Behr JP: Systemic linear polyethylenimine (L-PEI)-mediated gene delivery in the mouse. J Gene Med 2: 128-134, 2000.

13. Dalby B, Cates S, Harris A, et al: Advanced transfection with Lipofectamine 2000 reagent: Primary neurons, siRNA, and high-throughput applications. Methods 33: 95-103, 2004.

14. Kichler A, Leborgne C, Coeytaux E and Danos O: Polyethylenimine-mediated gene delivery: A mechanistic study. J Gene Med 3: 135-144, 2001.

15. Midoux P and Monsigny M: Efficient gene transfer by histidylated polylysine/pdna complexes. Bioconjug Chem 10: 406-411, 1999

16. Park YJ, Liang JF, Ko KS, Kim SW and Yang VC: Low molecular weight protamine as an efficient and nontoxic gene carrier: In vitro study. J Gene Med 5: 700-711, 2003.

17. Sun $X$ and Zhang ZR: Preparation of lipid-protamine-DNA complexes and evaluation of their transfection efficiencies in vitro. Yao Xue Xue Bao 39: 792-796, 2004.

18. Kircheis R, Wightman L and Wagner E: Design and gene delivery activity of modified polyethylenimines. Adv Drug Deliv Rev 53: 341-358, 2001.

19. Neu M, Fischer D and Kissel T: Recent advances in rational gene transfer vector design based on poly(ethylene imine) and its derivatives. J Gene Med 7: 992-1009, 2005.

20. Wadman M: Gene therapy might not have caused patient's death. Nature 449: 270, 2007.

21. Williams DA: ESCGT 2008: Progress in clinical gene therapy. Mol Ther 17: 1-2, 2009.

22. Ditto AJ, Shah PN and Yun YH: Non-viral gene delivery using nanoparticles. Expert Opin Drug Deliv 6: 1149-1160, 2009.

23. Pathak A, Patnaik S and Gupta KC: Recent trends in non-viral vector-mediated gene delivery. Biotechnol J 4: 1559-1572, 2009.

24. Tang GP and Lu X: [Progress in research on non-viral gene delivery vectors]. Zhejiang Da Xue Xue Bao Yi Xue Ban 38: 1-6, 2009.

25. Ewert KK, Ahmad A, Bouxsein NF, Evans HM and Safinya CR: Non-viral gene delivery with cationic liposome-DNA complexes. Methods Mol Biol 433: 159-175, 2008

26. Ishiwata H, Suzuki N, Ando S, Kikuchi H and Kitagawa T: Characteristics and biodistribution of cationic liposomes and their DNA complexes. J Control Release 69: 139-148, 2000.

27. Lv H, Zhang S, Wang B, Cui S and Yan J: Toxicity of cationic lipids and cationic polymers in gene delivery. J Control Release 114: 100-109, 2006.

28. Breunig M, Lungwitz U, Liebl R and Goepferich A: Breaking up the correlation between efficacy and toxicity for nonviral gene delivery. Proc Natl Acad Sci USA 104: 14454-14459, 2007.

29. Park TG, Jeong JH and Kim SW: Current status of polymeric gene delivery systems. Adv Drug Deliv Rev 58: 467-486, 2006.

30. Yamanouchi D, Wu J, Lazar AN, Kent KC, Chu CC and Liu B: Biodegradable arginine-based poly(ester-amide)s as non-viral gene delivery reagents. Biomaterials 29: 3269-3277, 2008.

31. Garcia L, Bunuales M, Duzgunes N and Tros de Ilarduya C: Serum-resistant lipopolyplexes for gene delivery to liver tumour cells. Eur J Pharm Biopharm 67: 58-66, 2007. 